Africa, and Prof. A. Aubréville similarly discussed the Sapotaceae.

Numerous other communications dealt with many topics more or less directly connected with taxonomy and plant distribution. A salutary reminder was given to the delegates by Prof. J. Léonard in an amusing discourse on the new African taxa published during the previous decade. His analysis showed the surprising facts that within this period an average of one genus per fortnight had been described and one species every day! The totals of all the new taxa, however, were remarkably constant, being about 2,500 in each of three three-year periods.

The second part of the conference was held in Florence. The delegates travelled from Genoa by road, stopping just south of Viareggio to examine the magnificent Pinus pinea forests and the coastal vegetation, which unfortunately is likely to be threatened by further encroach. ment of hotels and other seaside paraphernalia. The following day, September 13, an excursion was made into the spectacular Apuan Alps, from which much of the Italian marble is obtained, to see the interesting flora on these mountains.

In Florence the conference was resumed at the Istituto Botanico of the University at the invitation of the director, Prof. Eleonora Francini Corti, and Prof. Guido Moggi, curator of the herbarium. This herbarium is rich in plants from north-east tropical Africa. The botanical garden attached to the Institute possesses a yew tree (Taxus baccata) planted by Miquel about 1720, and delegates also had an opportunity to see the modern phytotron which is under the direction of Prof. Ornella Vergnano Gambi. During the final session, F. White gave a résumé of the thorough methods utilized by G. Prance during the latter's research on the genera of the Chrysobalanaceae. One was left in no doubt that modern taxonomy should be closely integrated with other disciplines in the field of botany. Other communications during this conference had already shown this tendency by African taxonomists in the preparation of Floras, but more monographic work is clearly desirable in order that information from subjects such as anatomy, cytology and the study of growth of seedlings can be taken into account for whole groups over their complete range.

The business meeting yielded important resolutions, among which it was proposed to publish in due course a revised edition of the Association's Vegetation Map of Africa South of the Sahara, and F. White (Oxford) was appointed secretary of the committee concerned with this. A further map is to be compiled showing the state of botanical exploration in Africa, and it is hoped to publish this together with the present conference proceedings, and to revise it at each successive conference. Dr. J. Leeuwenberg (Wageningen) announced that he was compiling an index of the location of duplicates of type specimens that were destroyed in the bombing of the Berlin Herbarium, and that he wished for the co-operation of members of the Association pour l'Etude Taxonomique de la Flore d'Afrique Tropicale.

There was also discussion of the fact that certain works dealing with African flora have been printed in insufficient numbers, but no definite decision was reached as to how this could be remedied.

The proceedings of the fifth conference are to be published in a fortheoming issue of Webbia. F. N. HEPPER

\title{
RESEARCH IN TEXTILES, COLOUR CHEMISTRY AND DYEING
}

$\mathrm{T}$ HE 1961-62 report to the Worshipful Company of Clothworkers of the Advisory Committee on the Departments of Textile Industries and Colour Chemistry and Dyeing in the University of Leeds* again records progress and achievement in both teaching and research. In the Department of Textile Industries, 67 of the 375 students in attendance were awarded higher degrees, postgraduate diplomas and degrees and 32 diplomas. Work on the quantitative aspects of the scattering of X-rays by keratin was delayed, but Dr. Sikorski's prediction of the mechanism involved in the formation of electron microscope images of transverse sections of keratin containing heavy elements was confirmed. It was also demonstrated that features comparable in dimensions with the resolving power of the modern electron microscope could be consistently obtained with some metals. The effect of crystallinity and crystalline orientation on the morphology of microfibrils in polyester, polyamide and polypropylene fibres was examined in some detail, and a method was discovered of identifying the amino-acid environment of the cross-linkages which are responsible for the increased modulus of wool fibres at extensions above 30 per cent, and of separating terylene from wool in admixture in the rags which form the raw material of the low woollen industry. In the study of the acid-base properties of fibrous materials, a new theory has been developed which treats the fibres of polyelectrolytes. Investigations into the constitution and properties of the by-products of the fleece continued, and the effect on dye. ing of scouring wool in the presence of organic solvents which facilitate low-temperature dyeing is being examined.

Measurements of the coefficient of friction of travellers under varying conditions have shown that for unlubri-

* Report to the Worshipful Company of Clothworkers of the City of London of the Advisory Committee on the Departments of Textile Indusries and Colour Chemistry and Dyeing in the University of Leeds for the Session 1961-1962. Pp. 48. (Leeds: The University, 1963.) cated travellers the coefficient of friction is correlated with a dimensionless group, which is proportional to the flash temperature of the surface; further investigation of the possibilities of high-draft woollen spinning has showa that the present limit cannot be exceeded. In textile technology, some aspects of cording are being investigated and a fundamental investigation was commenced of Noble spinning. Work on fibre-distribution in terylenecotton olivers, rovings and yarns was completed, and attention is being directed to the geometry of worn structures, with the view of obtaining information basic to the design of beat-up, take-up and let-off mechanisms. Research continued on the basic knitting section of the knitting machine; and, in finishing, research was directed towards obtaining fundamental information on the chemical and mechanical treatment of textile fibres and fabrics. Studies on wool were concerned mainly with the processes of milling and setting.

In the Department of Colour Chemistry and Dyeing there were 47 undergraduates and 18 research workers, and with Prof. Bradley's retirement the report gives some space to a summary of his research work in the Department since his appointment to the chair in 1948. During the year, work on the chlorination of anthroquinone in azocoupling in non-aqueous media was completed as well as that on $5: 6$-benzomesobenzanthrone and the chemistry of phenalones. Work was started on the effect of certain nucleophilic reagents on $2: 3$ dimethylphenalone, on the properties of 1-nitroanthroquinone-2-carboxylic chloride, with particular reference to dyes for acetate rayon, on the diffusion of dyes in single filaments and on the fading of pigments. Work continued on the mechanism of function of dioxazine dyes, the chemistry of $1: 1^{\prime}$-dinaphthyl and perylene and on the reactions of $9: 10$ benzomesobenzanthrone. Lists of publications are included in each of the departmental reports. 